\title{
Changing of the domination number of a graph: edge multisubdivision and edge removal
}

\author{
Vladimir Samodivkin \\ Department of Mathematics \\ University of Architecture Civil Engineering and Geodesy \\ Hristo Smirnenski 1 Blv., 1046 Sofia, Bulgaria, \\ vlsam_fte@uacg.bg
}

June 14, 2022

\begin{abstract}
For a graphical property $\mathcal{P}$ and a graph $G$, a subset $S$ of vertices of $G$ is a $\mathcal{P}$-set if the subgraph induced by $S$ has the property $\mathcal{P}$. The domination number with respect to the property $\mathcal{P}$, denoted by $\gamma_{\mathcal{P}}(G)$, is the minimum cardinality of a dominating $\mathcal{P}$-set. We define the domination multisubdivision number with respect to $\mathcal{P}$, denoted by $\operatorname{msd}_{\mathcal{P}}(G)$, as a minimum positive integer $k$ such that there exists an edge which must be subdivided $k$ times to change $\gamma_{\mathcal{P}}(G)$. In this paper (a) we present necessary and sufficient conditions for a change of $\gamma_{\mathcal{P}}(G)$ after subdividing an edge of $G$ once, (b) we prove that if $e$ is an edge of a graph $G$ then $\gamma_{\mathcal{P}}\left(G_{e, 1}\right)<\gamma_{\mathcal{P}}(G)$ if and only if $\gamma_{\mathcal{P}}(G-e)<\gamma_{\mathcal{P}}(G)\left(G_{e, t}\right.$ denote the graph obtained from $G$ by subdivision of $e$ with $t$ vertices), (c) we also prove that for every edge of a graph $G$ is fulfilled $\gamma_{\mathcal{P}}(G-e) \leq \gamma_{\mathcal{P}}\left(G_{e, 3}\right) \leq \gamma_{\mathcal{P}}(G-e)+1$, and (d) we show that $\operatorname{msd}_{\mathcal{P}}(G) \leq 3$, where $\mathcal{P}$ is hereditary and closed under union with $K_{1}$.
\end{abstract}

Keywords dominating set, edge subdivision, domination multisubdivision number, hereditary graph property

MSC 2010: 05C69 


\section{Introduction}

All graphs considered in this article are finite, undirected, without loops or multiple edges. For the graph theory terminology not presented here, we follow Haynes et al. 9]. We denote the vertex set and the edge set of a graph $G$ by $V(G)$ and $E(G)$, respectively. The subgraph induced by $S \subseteq V(G)$ is denoted by $\langle S, G\rangle$. For a vertex $x$ of $G, N(x, G)$ denotes the set of all neighbors of $x$ in $G, N[x, G]=N(x, G) \cup\{x\}$ and the degree of $x$ is $\operatorname{deg}(x, G)=|N(x, G)|$. The maximum and minimum degrees of vertices in the graph $G$ are denoted by $\Delta(G)$ and $\delta(G)$ respectively. For a graph $G$, let $x \in X \subseteq V(G)$. A vertex $y$ is a private neighbor of $x$ with respect to $X$ if $N[y, G] \cap X=\{x\}$. The private neighbor set of $x$ with respect to $X$ is $p n_{G}[x, X]=\{y: N[y, G] \cap X=\{x\}\}$. For a graph $G$ subdivision of the edge $e=u v \in E(G)$ with vertex $x$ leads to a graph with vertex set $V \cup\{x\}$ and edge set $(E-\{u v\}) \cup\{u x, x v\}$. Let $G_{e, t}$ denote graph obtained from $G$ by subdivision of the edge $e$ with $t$ vertices (instead of edge $e=u v$ we put a path $\left(u, x_{1}, x_{2}, \ldots, x_{t}, v\right)$ ). For $t=1$ we write $G_{e}$.

Let $\mathcal{I}$ denote the set of all mutually non-isomorphic graphs. A graph property is any non-empty subset of $\mathcal{I}$. We say that a graph $G$ has the property $\mathcal{P}$ whenever there exists a graph $H \in \mathcal{P}$ which is isomorphic to $G$. For example, we list some graph properties:

- $\mathcal{O}=\{H \in \mathcal{I}: H$ is totally disconnected $\}$

- $\mathcal{C}=\{H \in \mathcal{I}: H$ is connected $\}$;

- $\mathcal{T}=\{H \in \mathcal{I}: \delta(H) \geq 1\}$;

- $\mathcal{F}=\{H \in \mathcal{I}: H$ is a forest $\}$;

- $\mathcal{U} \mathcal{K}=\{H \in \mathcal{I}$ : each component of $H$ is complete $\}$;

- $\mathcal{D}_{k}=\{H \in \mathcal{I}: \Delta(H) \leq k\}$.

A graph property $\mathcal{P}$ is called: (a) hereditary (induced-hereditary), if from the fact that a graph $G$ has property $\mathcal{P}$, it follows that all subgraphs (induced subgraphs) of $G$ also belong to $\mathcal{P}$, and (b) nondegenerate if $\mathcal{O} \subseteq \mathcal{P}$. Any set $S \subseteq V(G)$ such that the induced subgraph $\langle S, G\rangle$ possesses the property $\mathcal{P}$ is called a $\mathcal{P}$ - set. Note that: (a) $\mathcal{I}, \mathcal{F}$ and $\mathcal{D}_{k}$ are nondegenerate and hereditary properties; (b) $\mathcal{U} \mathcal{K}$ is nondegenerate, induced-hereditary and is not hereditary; (c) both $\mathcal{C}$ and $\mathcal{T}$ are neither induced-hereditary nor nondegenerate. For a survey on this subject we refer to Borowiecki et al. [2].

A set of vertices $D \subseteq V(G)$ is a dominating set of a graph $G$ if every vertex not in $D$ is adjacent to a vertex in $D$. The domination number with respect to the property $\mathcal{P}$, denoted by $\gamma_{\mathcal{P}}(G)$, is the smallest cardinality of a dominating $\mathcal{P}$-set of $G$. A dominating $\mathcal{P}$-set of $G$ with cardinality $\gamma_{\mathcal{P}}(G)$ is called a $\gamma_{\mathcal{P}^{-}}$set of $G$. 
If a property $\mathcal{P}$ is nondegenerate, then every maximal independent set is a $\mathcal{P}$-set and thus $\gamma_{\mathcal{P}}(G)$ exists. Note that $\gamma_{\mathcal{I}}(G), \gamma_{\mathcal{O}}(G), \gamma_{\mathcal{C}}(G), \gamma_{\mathcal{T}}(G), \gamma_{\mathcal{F}}(G)$ and $\gamma_{\mathcal{D}_{k}}(G)$, are the well known as the domination number $\gamma(G)$, the independent domination number $i(G)$, the connected domination number $\gamma_{c}(G)$, the total domination number $\gamma_{t}(G)$, the acyclic domination number $\gamma_{a}(G)$ and the $k$-dependent domination number $\gamma^{k}(G)$, respectively (see [9]). The concept of domination with respect to any graph property $\mathcal{P}$ was introduced by Goddard et al. [5] and has been studied, for example, in [10, 11, 12, 13, 14, and elsewhere.

It is often of interest to know how the value of a graph parameter is affected when a small change is made in a graph. In [11], the present author began the study of the effects on $\gamma_{\mathcal{P}}(G)$ when a graph $G$ is modified by deleting a vertex or by adding an edge $(\mathcal{P}$ is nondegenerate). In this paper we concentrate on effects on $\gamma_{\mathcal{P}}(G)$ when a graph is modified by deleting/subdividing an edge. An edge $e$ of a graph $G$ is called a $\gamma_{\mathcal{P}}-E R^{-}$-critical edge of $G$ if $\gamma_{\mathcal{P}}(G)>\gamma_{\mathcal{P}}(G-e)$. Note that (a) there do not exist $\gamma-E R^{-}$-critical edges (see [8]), (b) Grobler [6] was the first who began the investigation of $\gamma_{\mathcal{P}^{-}} E R^{-}$-critical edges when $\mathcal{P}=\mathcal{O}$, and (c) necessary and sufficient conditions for an edge of a graph $G$ to be $\gamma_{\mathcal{H}}-E R^{-}$-critical may be found in [11].

When an edge of a graph $G$ is subdivided, the domination number with respect to the property $\mathcal{P}$ can increase or decrease. For instance, if $G$ is a star $K_{1, p}, p \geq 2$, and $\left\{K_{1}, 2 K_{1}\right\} \subseteq \mathcal{P} \subseteq \mathcal{I}$ then $\gamma_{\mathcal{P}}(G)=1$ and $\gamma_{\mathcal{P}}\left(G_{e}\right)=2$ for all $e$. If a graph $G$ is obtained by three stars $K_{1, p}$ and three edges $e_{1}, e_{2}, e_{3}$ joining their centers then $\gamma_{\mathcal{F}}(G)=2+p$ and $\gamma_{\mathcal{F}}\left(G_{e_{i}}\right)=3, i=1,2,3$. This motivates the next definitions.

For any nondegenerate property $\mathcal{P} \subseteq \mathcal{I}$ we define the edge $e$ of a graph $G$ to be (a) a $\gamma_{\mathcal{P}}-S^{+}$-critical edge of $G$ if $\gamma_{\mathcal{P}}(G)<\gamma_{\mathcal{P}}\left(G_{e}\right)$, and (b) a $\gamma_{\mathcal{P}}-S^{-}$-critical edge of $G$ if $\gamma_{\mathcal{P}}(G)>\gamma_{\mathcal{P}}\left(G_{e}\right)$. In Section 2: (a) we present necessary and sufficient conditions for a change of $\gamma_{\mathcal{P}}(G)$ after subdividing an edge of $G$ once, and (b) we prove that an edge $e$ of a graph $G$ is $\gamma_{\mathcal{H}^{-}} \mathrm{S}^{-}$-critical if and only if $e$ is $\gamma_{\mathcal{H}^{-}} E R^{-}$-critical, where $\mathcal{H} \subseteq \mathcal{I}$ is any induced-hereditary and closed under union with $K_{1}$ graph property.

In Section 3 we deals with changing of $\gamma_{\mathcal{P}}(G)$ when an edge of $G$ is multiple subdivided. To present our results we need the following definitions.

For every edge $e$ of a graph $G$ let

- $\operatorname{msd}_{\mathcal{P}}(e)=\min \left\{t \mid \gamma_{\mathcal{P}}\left(G_{e, t}\right) \neq \gamma_{\mathcal{P}}(G)\right\}$;

- $\operatorname{msd}_{\mathcal{P}}^{+}(e)=\min \left\{t \mid \gamma_{\mathcal{P}}\left(G_{e, t}\right)>\gamma_{\mathcal{P}}(G)\right\}$

- $\operatorname{msd}_{\mathcal{P}}^{-}(e)=\min \left\{t \mid \gamma_{\mathcal{P}}\left(G_{e, t}\right)<\gamma_{\mathcal{P}}(G)\right\}$.

If $\gamma_{\mathcal{P}}\left(G_{e, t}\right) \geq \gamma_{\mathcal{P}}(G)$ for every $t, t \geq 1$, then we write $\operatorname{msd}_{\mathcal{P}}^{-}(e)=\infty$. If $\gamma_{\mathcal{P}}\left(G_{e, t}\right) \leq \gamma_{\mathcal{P}}(G)$ for every $t, t \geq 1$, then we write $\operatorname{msd}_{\mathcal{P}}^{+}(e)=\infty$.

For every graph $G$ with at least one edge and every nondegenerate property $\mathcal{P}$, 
we define:

$\left(\mathbf{D}_{1}\right)$ the domination multisubdivision (plus domination multisubdivision, minus domination multisubdivision) number with respect to the property $\mathcal{P}$, denoted $m s d_{\mathcal{P}}(G)\left(m s d_{\mathcal{P}}^{+}, m s d_{\mathcal{P}}^{-}(G)\right.$, respectively) to be

- $\operatorname{msd}_{\mathcal{P}}(G)=\min \left\{\operatorname{msd}_{\mathcal{P}}(e) \mid e \in E(G)\right\}$,

- $m s d_{\mathcal{P}}^{+}(G)=\min \left\{m s d_{\mathcal{P}}^{+}(e) \mid e \in E(G)\right\}$, and

- $m s d_{\mathcal{P}}^{-}(G)=\min \left\{m s d_{\mathcal{P}}^{-}(e) \mid e \in E(G)\right\}$,

respectively. If $\gamma_{\mathcal{P}}\left(G_{e, t}\right) \geq \gamma_{\mathcal{P}}(G)$ for every $t$, and every edge $e \in E(G)$, then we write $\operatorname{msd}_{\mathcal{P}}^{-}(G)=\infty$.

The parameters $m s d^{+}(G)$ and $m s d_{\mathcal{T}}^{+}(G)$ (in our designation) was introduced by Dettlaff, Raczek and Topp in [4] and by Avella-Alaminos, Dettlaff, Lemańska and Zuazua [1, respectively. Note that in the case when $\mathcal{P}=\mathcal{I}$, clearly, $\operatorname{msd}(G)=$ $\mathrm{msd}^{+}(G)$, and $\mathrm{msd}^{-}(G)=\infty$. In Section 3 we prove that for every edge of a graph $G$ is fulfilled $\gamma_{\mathcal{P}}(G-e) \leq \gamma_{\mathcal{P}}\left(G_{e, 3}\right) \leq \gamma_{\mathcal{P}}(G-e)+1$ and we present necessary and sufficient conditions for the validity of $\gamma_{\mathcal{P}}(G-e)=\gamma_{\mathcal{P}}\left(G_{e, 3}\right)$. Our main result in this section is that $\operatorname{msd}_{\mathcal{P}}(G) \leq 3$ for any graph $G$ and any hereditary and closed under union with $K_{1}$ graph-property $\mathcal{P}$.

\section{Single subdivision: critical edges}

We begin this section with a characterization of $\gamma_{\mathcal{P}}-S^{+}$-critical edges of a graph. Note that if a property $\mathcal{P}$ is induced-hereditary and closed under union with $K_{1}$ then $\mathcal{P}$ is nondegenerate.

Theorem 2.1. Let $\mathcal{H} \subseteq \mathcal{I}$ be hereditary and closed under union with $K_{1}$. Let $G$ be a graph and $e=u v \in E(G)$. Then $\gamma_{\mathcal{H}}\left(G_{e}\right) \leq \gamma_{\mathcal{H}}(G)+1$. If e is a $\gamma_{\mathcal{H}}{ }^{-} S^{+}$-critical edge of $G$ then $\gamma_{\mathcal{H}}\left(G_{e}\right)=\gamma_{\mathcal{H}}(G)+1$ and for each $\gamma_{\mathcal{H}}$-set $M$ of $G$ one of the following holds:

(i) $u, v \in V(G)-M$;

(ii) $u \in M, v \in p n_{G}[u, M]$ and $p n_{G}[u, M]$ is not a subset of $\{u, v\}$;

(iii) $v \in M, u \in p n_{G}[v, M]$ and $p n_{G}[u, M]$ is not a subset of $\{u, v\}$.

If e is not $\gamma_{\mathcal{P}}-S^{+}$-critical and for each $\gamma_{\mathcal{H}}$-set $M$ of $G$ one of (i), (ii) and (iii) holds then there is a dominating $\mathcal{H}$-set $R$ of $G-u v$ with $u, v \in R$ and $|R| \leq \gamma_{\mathcal{H}}(G)$.

Proof. Let $x \in V\left(G_{e}\right)$ be the subdivision vertex and let $M$ be a $\gamma_{\mathcal{H}}$-set of $G$. If $u, v \notin M$ then $M \cup\{x\}$ is a dominating $\mathcal{H}$-set of $G_{e}(\mathcal{H}$ is closed under union 
with $\left.K_{1}\right)$ and we have $\gamma_{\mathcal{H}}\left(G_{e}\right) \leq \gamma_{\mathcal{H}}(G)+1$. If both $u$ and $v$ are in $M$ then $M$ is a dominating $\mathcal{H}$-set of $G_{e}\left(\mathcal{H}\right.$ is hereditary) which implies $\gamma_{\mathcal{H}}\left(G_{e}\right) \leq \gamma_{\mathcal{H}}(G)$. If $u \in M, v \notin M$ and $v \notin p n_{G}[u, M]$ then again $M$ is a dominating $\mathcal{H}$-set of $G_{e}$ and hence $\gamma_{\mathcal{H}}\left(G_{e}\right) \leq \gamma_{\mathcal{H}}(G)$. So, let $u \in M, v \notin M$ and $v \in p n_{G}[u, M]$. If either $\{v\}$ or $\{u, v\}$ coincides with $p n_{G}[u, M]$ then $(M-\{u\}) \cup\{x\}$ is a dominating $\mathcal{H}$-set of $G_{e}$; hence $\gamma_{\mathcal{H}}\left(G_{e}\right) \leq \gamma_{\mathcal{H}}(G)$. If neither $p n_{G}[u, M]=\{v\}$ nor $p n_{G}[u, M]=\{u, v\}$ then $M \cup\{v\}$ is a dominating $\mathcal{H}$-set of $G_{e}$ and we have $\gamma_{\mathcal{H}}\left(G_{e}\right) \leq \gamma_{\mathcal{H}}(G)+1$. Thus $\gamma_{\mathcal{H}}\left(G_{e}\right) \leq \gamma_{\mathcal{H}}(G)+1$ and if the equality is fulfilled then one of (i), (ii) and (iii) holds.

Now, let for each $\gamma_{\mathcal{H}}$-set $M$ of $G$ one of (i), (ii) and (iii) hold. Assume $\gamma_{\mathcal{H}}\left(G_{e}\right) \leq$ $\gamma_{\mathcal{H}}(G)$ and let $R$ be a $\gamma_{\mathcal{H}}$-set of $G_{e}$.

Case 1: $u, v \notin R$. Hence $x \in R$. If $u, v \notin p n_{G_{e}}[x, R]$ then $R-\{x\}$ is a dominating $\mathcal{H}$-set of $G$, a contradiction with $\gamma_{\mathcal{H}}\left(G_{e}\right) \leq \gamma_{\mathcal{H}}(G)$. If $u \in p n_{G_{e}}[x, R]$ and $v \notin p n_{G_{e}}[x, R]$ then $R_{1}=(R-\{x\}) \cup\{u\}$ is a dominating $\mathcal{H}$-set of $G$ of cardinality $\left|R_{1}\right|=|R|=\gamma_{\mathcal{H}}\left(G_{e}\right)$. Since $\gamma_{\mathcal{H}}\left(G_{e}\right) \leq \gamma_{\mathcal{H}}(G)$, we have that $R_{1}$ is a $\gamma_{\mathcal{H}^{-}}$set of $G$. But then $u \in R_{1}, v \notin R_{1}$ and $v \notin p n_{G}\left[u, R_{1}\right]$, contradicting (ii). If $u, v \in p n_{G}[x, R]$ then as above $R_{1}$ is a $\gamma_{\mathcal{H}^{-}}$set of $G$ and since $u \in R_{1}$ and $\{u, v\}=p n_{G}\left[u, R_{1}\right]$, again we arrive to a contradiction with (ii).

Case 2: $u \in R$ and $v \notin R$. Hence $x \notin R$, otherwise $R-\{x\}$ is a dominating $\mathcal{H}$-set of $G$, contradicting $\gamma_{\mathcal{H}}\left(G_{e}\right) \leq \gamma_{\mathcal{H}}(G)$. This implies that $R$ is a $\gamma_{\mathcal{H}}$-set of $G$, $u \in R$ and $v \notin p n_{G}[u, R]$, a contradiction with (ii).

Case 3: $u, v \in R$. Hence $R$ is a dominating $\mathcal{H}$-set of $G-u v$ and $|R|=\gamma_{\mathcal{H}}\left(G_{e}\right) \leq$ $\gamma_{\mathcal{H}}(G)$.

When we restrict our attention to the case where $\mathcal{H}=\mathcal{I}$, we can describe more precisely when an edge of a graph $G$ is $\gamma-\mathrm{S}^{+}$-critical.

Corollary 2.2. Let $G$ be a graph and $e=u v \in E(G)$. Then e is a $\gamma$-S $S^{+}$-critical edge of $G$ if and only if for each $\gamma$-set $M$ of $G$ one of (i), (ii) and (iii) stated in Theorem 2.1 holds.

Proof. Necessity: The result immediately follows by Theorem 2.1.

Sufficiency: Assume $\gamma\left(G_{e}\right) \leq \gamma(G)$. Then by Theorem 2.1, there is a dominating set $R$ of $G-u v$ with $u, v \in R$ and $|R| \leq \gamma(G)$. But it is well known fact that if $f$ is an edge of a graph $G$ then always $\gamma(G-f) \geq \gamma(G)$. Hence $R$ is a $\gamma$-set of both $G$ and $G-e$ and $u, v \in R$, contradicting all (i), (ii) and (iii).

Theorem 2.3. Let $\mathcal{H} \subseteq \mathcal{I}$ be induced-hereditary and closed under union with $K_{1}$. An edge e of a graph $G$ is $\gamma_{\mathcal{H}}{ }^{-}{ }^{-}$-critical if and only if $e$ is $\gamma_{\mathcal{H}}-E R^{-}$-critical. 
Proof. As we have already know, $\mathcal{H}$ is nondegenerate and then all $\gamma_{\mathcal{H}}(G-e)$, $\gamma_{\mathcal{H}}\left(G_{e}\right)$ and $\gamma_{\mathcal{H}}(G)$ exist. Let $v$ be the subdivision vertex of $G_{e}$.

Sufficiency: Let $e=x y$ be a $\gamma_{\mathcal{H}^{-}} E R^{-}$-critical edge of $G$ and $M$ a $\gamma_{\mathcal{H}^{-}}$-set of $G-e$. Hence $\gamma_{\mathcal{H}}(G-e)<\gamma_{\mathcal{H}}(G)$ and $x, y \in M$. But then $M$ is a dominating $\mathcal{H}$-set of $G_{e}$ which leads to $\gamma_{\mathcal{H}}\left(G_{e}\right) \leq \gamma_{\mathcal{H}}(G-e)<\gamma_{\mathcal{H}}(G)$.

Necessity: Let $e=x y$ be a $\gamma_{\mathcal{H}^{-}} \mathrm{S}^{-}$-critical edge of $G$ and $M$ a $\gamma_{\mathcal{H}}$-set of $G_{e}$. Hence $\gamma_{\mathcal{H}}\left(G_{e}\right)<\gamma_{\mathcal{H}}(G)$. Assume $v \notin M$. Hence at least one of $x$ and $y$ is in $M$. If both $x, y \in M$ then $M$ is a dominating $\mathcal{H}$-set of $G-e$ and the result follows. If $x \notin M$ and $y \in M$ then $M$ is a dominating $\mathcal{H}$-set of $G$, a contradiction. Thus we may assume $v$ is in all $\gamma_{\mathcal{H}}$-sets of $G_{e}$. Since $\mathcal{H}$ is induced-hereditary, at least one of $x$ and $y$ is not in $M$. First let $x \in M$ and $y \notin M$. Then $y \in p n_{G_{e}}[v, M]$ which implies $M-\{v\}$ is a dominating $\mathcal{H}$-set of $G$ - a contradiction. Hence both $x$ and $y$ are not in $M$. If $x, y \notin p n_{G_{e}}[v, M]$ then $M-\{v\}$ is a dominating $\mathcal{H}$-set of $G$, a contradiction. Hence at least one of $x$ and $y$, say $y$, is in $p n_{G_{e}}[v, M]$. But then $(M-\{v\}) \cup\{y\}$ is a dominating $\mathcal{H}$-set of $G$, a contradiction.

Note that (a) there do not exist $\gamma-E R^{-}$-critical edges (see [8]), and (b) necessary and sufficient conditions for an edge of a graph $G$ to be $\gamma_{\mathcal{H}^{-}} E R^{-}$-critical may be found in [11]. Now we define the following classes of graphs:

- $\left(C S_{\mathcal{P}}^{-}\right) \gamma_{\mathcal{P}}(G)>\gamma_{\mathcal{P}}\left(G_{e}\right)$ for every edge $e$ of $G$, and

- $\left(C E R_{\mathcal{P}}^{-}\right) \gamma_{\mathcal{P}}(G)>\gamma_{\mathcal{P}}(G-e)$ for every edge $e$ of $G$.

As an immediate consequence of Theorem 2.3 we obtain the next result.

Corollary 2.4. If $\mathcal{H} \subseteq \mathcal{I}$ is induced-hereditary and closed under union with $K_{1}$ then the classes of graphs $\left(C S_{\mathcal{P}}^{-}\right)$and $\left(C E R_{\mathcal{P}}^{-}\right)$coincide.

Note that the class $\left(C E R_{\mathcal{P}}^{-}\right)$in the case when $\mathcal{P}=\mathcal{O}$ was introduced by Grobler [6] and also considered in [7, 8, [3].

\section{Multiple subdivision}

Recall that $G_{e, t}$ denote the graph obtained from a graph $G$ by subdivision of the edge $e \in E(G)$ with $t$ vertices (instead of edge $e=u v$ we put a path $\left(u, x_{1}, x_{2}, \ldots, x_{t}, v\right)$ ). For any graph $G$ and any nondegenerate property $\mathcal{P}$ let us denote by $V_{\mathcal{P}}^{-}(G)$ the set $\left\{v \in V(G) \mid \gamma_{\mathcal{P}}(G-v)<\gamma_{\mathcal{P}}(G)\right\}$. Qur first result shows that the value of the difference $\gamma_{\mathcal{P}}\left(G_{e, 3}\right)-\gamma_{\mathcal{P}}(G-e)$ can be either 0 or 1 .

Theorem 3.1. Let $\mathcal{H} \subseteq \mathcal{I}$ be induced-hereditary and closed under union with $K_{1}$. If $e=u v$ is an edge of a graph $G$ then $\gamma_{\mathcal{H}}(G-e) \leq \gamma_{\mathcal{H}}\left(G_{e, 3}\right) \leq \gamma_{\mathcal{H}}(G-e)+1$. Moreover, the following conditions are equivalent: 
$\left(\mathbb{A}_{1}\right) \gamma_{\mathcal{H}}(G-e)=\gamma_{\mathcal{H}}\left(G_{e, 3}\right)$;

$\left(\mathbb{A}_{2}\right)$ at least one of the following holds:

(i) $u \in V_{\mathcal{H}}^{-}(G-e)$ and $v$ belongs to some $\gamma_{\mathcal{H}}$-set of $G-u$;

(ii) $v \in V_{\mathcal{H}}^{-}(G-e)$ and $u$ belongs to some $\gamma_{\mathcal{H}}$-set of $G-v$.

If in addition $\mathcal{H}$ is hereditary then $\left(\mathbb{A}_{1}\right)$ and $\left(\mathbb{A}_{2}\right)$ are equivalent to

$\left(\mathbb{A}_{3}\right) \gamma_{\mathcal{H}}(G-e)=1+\gamma_{\mathcal{H}}(G)$.

The main result in this section is the following.

Theorem 3.2. Let $e$ be an edge of a graph $G$ and let $\mathcal{H} \subseteq \mathcal{I}$ be hereditary and closed under union with $K_{1}$.

(i) Then $\gamma_{\mathcal{H}}(G)=\gamma_{\mathcal{H}}\left(G_{e, 3}\right)$ if and only if $\gamma_{\mathcal{H}}(G)=\gamma_{\mathcal{H}}(G-e)+1$.

(ii) If $\gamma_{\mathcal{H}}(G)=\gamma_{\mathcal{H}}(G-e)+1$ then $\operatorname{msd}_{\mathcal{H}}(e)=\operatorname{msd}_{\mathcal{H}}^{-}(e)=1, \operatorname{msd}_{\mathcal{H}}^{+}(e)=6$ and $\gamma_{\mathcal{H}}(G)=\gamma_{\mathcal{H}}\left(G_{e, 1}\right)+1=\gamma_{\mathcal{H}}\left(G_{e, 2}\right)+1=\gamma_{\mathcal{H}}\left(G_{e, 3}\right)=\gamma_{\mathcal{H}}\left(G_{e, 4}\right)=\gamma_{\mathcal{H}}\left(G_{e, 5}\right)=$ $\gamma_{\mathcal{H}}\left(G_{e, 6}\right)-1$.

(iii) Then $\operatorname{msd}_{\mathcal{H}}(e) \leq 3$. In particular, (Dettlaff, Raczek and Topp [4] when $\mathcal{H}=$ I) $\operatorname{msd}_{\mathcal{H}}(G) \leq 3$.

Example 3.3. It is easy to see that if $G=K_{3 n_{2} . . n_{m}}$, where $m \geq 2$ and $n_{i} \geq 3$ for $2 \leq i \leq m$, then $\gamma_{\mathcal{O}}(G)=\gamma_{\mathcal{O}}\left(G_{e, 3}\right)=\gamma_{\mathcal{O}}(G-e)+1=3$ for every edge e of $G$. Hence by Theorem 3.2, $\operatorname{msd}_{\mathcal{O}}(G)=\operatorname{msd}_{\mathcal{O}}^{-}(G)=1$ and $\operatorname{msd}_{\mathcal{O}}^{+}(G)=6$.

In view of Theorem 3.2(iii), we can split the family of all graphs $G$ into three classes with respect to the value of $\operatorname{msd}_{\mathcal{P}}(G)$, where $\mathcal{P} \subseteq \mathcal{I}$ is hereditary and closed under union with $K_{1}$. We define that a graph $G$ belongs to the class $S_{\mathcal{P}}^{i}$ whenever $\operatorname{msd}_{\mathcal{P}}(G)=i, i \in\{1,2,3\}$. It is straightforward to verify that if $k \geq 1$ and $\mathcal{O} \subseteq \mathcal{P} \subseteq \mathcal{I}$ then

- $P_{3 k}, C_{3 k} \in S_{\mathcal{P}}^{1} ; P_{3 k+2}, C_{3 k+2} \in S_{\mathcal{P}}^{2} ;$ and $P_{3 k+1}, C_{3 k+1} \in S_{\mathcal{P}}^{3}$.

Thus, none of $S_{\mathcal{P}}^{1}, S_{\mathcal{P}}^{2}$ and $S_{\mathcal{P}}^{3}$ is empty.

We conclude this part with an open problem.

Problem 3.4. Characterize the graphs belonging to $S_{\mathcal{P}}^{i}$, or find further properties of such graphs.

Remark that Dettlaff, Raczek and Topp recently characterized all trees belonging to $S^{1}$ and $S^{3}$ (see [4]). 


\subsection{Proofs}

For the proofs of Theorems 3.1 and 3.1, we need the following results.

Theorem A ([1] ). Let $\mathcal{H} \subseteq \mathcal{I}$ be nondegenerate and closed under union with $K_{1}$. Let $G$ be a graph and $v \in V(G)$.

(i) If $v$ belongs to no $\gamma_{\mathcal{H}}$-set of $G$ then $\gamma_{\mathcal{H}}(G-v)=\gamma_{\mathcal{H}}(G)$.

(ii) If $\gamma_{\mathcal{H}}(G-v)<\gamma_{\mathcal{H}}(G)$ then $\gamma_{\mathcal{H}}(G-v)=\gamma_{\mathcal{H}}(G)-1$. Moreover, if $M$ is a $\gamma_{\mathcal{H}^{-}}$set of $G-v$ then $M \cup\{v\}$ is a $\gamma_{\mathcal{H}}$-set of $G$ and $\{v\}=p n_{G}[v, M \cup\{v\}]$.

Theorem B ([11]). Let $\mathcal{H} \subseteq \mathcal{I}$ be hereditary and closed under union with $K_{1}$. Let $e=u v$ be an edge of a graph $G$. If $\gamma_{\mathcal{H}}(G)<\gamma_{\mathcal{H}}(G-e)$ then $\gamma_{\mathcal{H}}(G)=\gamma_{\mathcal{H}}(G-e)-1$. Moreover $\gamma_{\mathcal{H}}(G)=\gamma_{\mathcal{H}}(G-e)-1$ if and only if at least one of the conditions (i) and (ii) stated in Theorem 3.1 holds.

Theorem $\mathbf{C}([11])$. Let $e=x y$ be an edge of a graph $G$ and let $\mathcal{H} \subseteq \mathcal{I}$ be hereditary and closed under union with $K_{1}$. If $\gamma_{\mathcal{H}}(G)>\gamma_{\mathcal{H}}(G-e)$ then:

(i) no $\gamma_{\mathcal{H}}$-set of $G-e$ is an $\mathcal{H}$-set of $G$;

(ii) both $x$ and $y$ are in all $\gamma_{\mathcal{H}}$-sets of $G-e$;

(iii) $\gamma_{\mathcal{H}}(G-x) \geq \gamma_{\mathcal{H}}(G-e)$ and $\gamma_{\mathcal{H}}(G-y) \geq \gamma_{\mathcal{H}}(G-e)$;

(iv) if $\gamma_{\mathcal{H}}(G-x)=\gamma_{\mathcal{H}}(G-e)$ then $y$ belongs to no $\gamma_{\mathcal{H}}$-set of $G-x$;

(v) if $\gamma_{\mathcal{H}}(G-y)=\gamma_{\mathcal{H}}(G-e)$ then $x$ belongs to no $\gamma_{\mathcal{H}}$-set of $G-y$.

Proof of Theorem 3.1. Let $D$ be a $\gamma_{\mathcal{H}}$-set of $G-e$. Then since $\mathcal{H}$ is closed under union with $K_{1}, D \cup\left\{x_{2}\right\}$ is a dominating $\mathcal{H}$-set of $G_{e, 3}$. Hence $\gamma_{\mathcal{H}}\left(G_{e, 3}\right) \leq$ $|D \cup\{y\}| \leq \gamma_{\mathcal{H}}(G-e)+1$.

For the left side inequality, let $\widetilde{D}$ be a $\gamma_{\mathcal{H}}$-set of $G_{e, 3}$ and $S=\widetilde{D} \cap\left\{x_{1}, x_{2}, x_{3}\right\}$. If $S=\left\{x_{2}\right\}$ then $\widetilde{D}-\left\{x_{2}\right\}$ is a dominating $\mathcal{H}$-set of $G-e$ and $\gamma_{\mathcal{H}}(G-e) \leq$ $\left|\widetilde{D}-\left\{x_{2}\right\}\right|=\gamma_{\mathcal{H}}\left(G_{e, 3}\right)-1$. If $S=\left\{x_{1}, x_{2}\right\}$ then $p n_{G_{e, 3}}\left[x_{1}, \widetilde{D}\right]=\{u\}$ and hence $\widetilde{D}_{1}=\left(\widetilde{D}-\left\{x_{1}, x_{2}\right\}\right) \cup\{u\}$ is a dominating $\mathcal{H}$-set of $G-e$ which implies $\gamma_{\mathcal{H}}(G-e) \leq$ $\left|\widetilde{D}_{1}\right|<|\widetilde{D}|=\gamma_{\mathcal{H}}\left(G_{e, 3}\right)$.

Let $S=\left\{x_{1}\right\}$. If $u \in \operatorname{pn}\left[x_{1}, \widetilde{D}\right]$ then $\widetilde{D}_{2}=\left(\widetilde{D}-\left\{x_{1}\right\}\right) \cup\{u\}$ is a dominating $\mathcal{H}$-set of $G-e$ and hence $\gamma_{\mathcal{H}}(G-e) \leq\left|\widetilde{D}_{2}\right|=|\widetilde{D}|=\gamma_{\mathcal{H}}\left(G_{e, 3}\right)$. If $u \notin p n\left[x_{1}, \widetilde{D}\right]$ then $\widetilde{D}-\left\{x_{1}\right\}$ is a dominating $\mathcal{H}$-set of $G-e$ and $\gamma_{\mathcal{H}}(G-e) \leq|\widetilde{D}|-1=\gamma_{\mathcal{H}}\left(G_{e, 3}\right)-1$. 
If $S=\left\{x_{1}, x_{3}\right\}$ then at least one of $p n_{G_{e, 3}}\left[x_{1}, \widetilde{D}\right]=\left\{x_{1}, u\right\}$ and $p n_{G_{e, 3}}\left[x_{3}, \widetilde{D}\right]=$ $\left\{x_{3}, v\right\}$ holds, otherwise $\left(\widetilde{D}-\left\{x_{1}, x_{3}\right\}\right) \cup\left\{x_{2}\right\}$ would be a dominating $\mathcal{H}$-set of $G_{e, 3}$, contradicting the choice of $\widetilde{D}$. Say, without loss of generality, $p n_{G_{e, 3}}\left[x_{3}, \widetilde{D}\right]=$ $\left\{x_{3}, v\right\}$. Then $\widetilde{D}_{3}=\left(\widetilde{D}-\left\{x_{3}\right\}\right) \cup\{v\}$ is a $\gamma_{\mathcal{H}}$-set of $G_{e, 3}$ and $\widetilde{D}_{3} \cap\left\{x_{1}, x_{2}, x_{3}\right\}=\left\{x_{1}\right\}$. As above we obtain $\gamma_{\mathcal{H}}(G-e)<\gamma_{\mathcal{H}}\left(G_{e, 3}\right)$. By reason of symmetry, the left side inequality is proved.

$\left(\mathbb{A}_{2}\right) \Rightarrow\left(\mathbb{A}_{1}\right)$ Assume without loss of generality that (i) holds. Let $D$ be a $\gamma_{\mathcal{H}}(G-u)$-set and $v \in D$. By Theorem $\square$ A, $D \cup\{u\}$ is a $\gamma_{\mathcal{H}}$-set of $G-e$ and $p n_{G-e}[u, D \cup\{u\}]=\{u\}$. Hence $D \cup\left\{x_{1}\right\}$ is a dominating $\mathcal{H}$-set of $G_{e, 3}$ and $\gamma_{\mathcal{H}}\left(G_{e, 3}\right) \leq\left|D \cup\left\{x_{1}\right\}\right|=\gamma_{\mathcal{H}}(G-e)$. But we have already known that $\gamma_{\mathcal{H}}\left(G_{e, 3}\right) \geq$ $\gamma_{\mathcal{H}}(G-e)$. Therefore $\gamma_{\mathcal{H}}\left(G_{e, 3}\right)=\gamma_{\mathcal{H}}(G-e)$.

$\left(\mathbb{A}_{1}\right) \Rightarrow\left(\mathbb{A}_{2}\right)$ Suppose $\gamma_{\mathcal{H}}(G-e)=\gamma_{\mathcal{H}}\left(G_{e, 3}\right)$. Let $\widetilde{D}$ be a $\gamma_{\mathcal{H}}\left(G_{e, 3}\right)$-set and $S=\widetilde{D} \cap\left\{x_{1}, x_{2}, x_{3}\right\}$. If $S=\left\{x_{2}\right\}$ then $\widetilde{D}-\left\{x_{2}\right\}$ is a dominating $\mathcal{H}$-set of $G-e$, a contradiction. If $S=\left\{x_{1}, x_{2}\right\}$ then clearly $p n_{G_{e, 3}}\left[x_{1}, \widetilde{D}\right]=\{u\}$ which implies that $\left(\widetilde{D}-\left\{x_{1}, x_{2}\right\}\right) \cup\{u\}$ is a dominating $\mathcal{H}$-set of $G-e$, a contradiction.

Let $S=\left\{x_{1}\right\}$. Hence $v \in \widetilde{D}$. If $u \notin p n_{G_{e, 3}}\left[x_{1}, \widetilde{D}\right]$ then $\widetilde{D}-\left\{x_{1}\right\}$ is a dominating $\mathcal{H}$-set of $G-e$, a contradiction. If $u \in p n_{G_{e, 3}}\left[x_{1}, \widetilde{D}\right]$ then $D_{1}=\left(\widetilde{D}-\left\{x_{1}\right\}\right) \cup\{u\}$ is a $\gamma_{\mathcal{H}}$-set of $G-e, u, v \in D_{1}, D_{1}-\{u\}$ is a $\gamma_{\mathcal{H}}$-set of $G-u$ (by Theorem $\mathrm{A}$ ) and $v \in D_{1}-\{u\}$. In addition it follows that $u \in V_{\mathcal{H}}^{-}(G-e)$. Thus, (i) holds.

By symmetry it remains the case when $S=\left\{x_{1}, x_{3}\right\}$. If $u \notin p n_{G_{e, 3}}\left[x_{1}, \widetilde{D}\right]$ and $v \notin p n_{G_{e, 3}}\left[x_{3}, \widetilde{D}\right]$ then $\widetilde{D}-\left\{x_{1}, x_{3}\right\}$ is a dominating $\mathcal{H}$-set of $G-e$, a contradiction. If $u \in p n_{G_{e, 3}}\left[x_{1}, \widetilde{D}\right]$ and $v \notin p n_{G_{e, 3}}\left[x_{3}, \widetilde{D}\right]$ then $\left(\widetilde{D}-\left\{x_{1}, x_{3}\right\}\right) \cup\{u\}$ is a dominating $\mathcal{H}$-set of $G-e$, a contradiction. So, $u \in p n_{G_{e, 3}}\left[x_{1}, \widetilde{D}\right]$ and $v \in p n_{G_{e, 3}}\left[x_{3}, \widetilde{D}\right]$. Then $D_{2}=\left(\widetilde{D}-\left\{x_{1}, x_{3}\right\}\right) \cup\{u, v\}$ is a $\gamma_{\mathcal{H}}$-set of $G-e$ and both $\{u\}=p n_{G-e}\left[x_{1}, D_{2}\right]$ and $\{v\}=p n_{G-e}\left[x_{3}, D_{2}\right]$ hold. Thus both (i) and (ii) are fulfilled.

\section{$\left(\mathbb{A}_{2}\right) \Leftrightarrow\left(\mathbb{A}_{3}\right)$ By Theorem $\mathrm{B}$,}

Proof of Theorem 3.2. (i) Necessity: Let $\gamma_{\mathcal{H}}(G)=\gamma_{\mathcal{H}}\left(G_{e, 3}\right)$. By Theorem 3.1 we know that $\gamma_{\mathcal{H}}(G-e) \leq \gamma_{\mathcal{H}}\left(G_{e, 3}\right) \leq \gamma_{\mathcal{H}}(G-e)+1$ and if $\gamma_{\mathcal{H}}(G-e)=\gamma_{\mathcal{H}}\left(G_{e, 3}\right)$ then $\gamma_{\mathcal{H}}\left(G_{e, 3}\right)=\gamma_{\mathcal{H}}(G)+1$. Thus $\gamma_{\mathcal{H}}(G)=\gamma_{\mathcal{H}}\left(G_{e, 3}\right)=\gamma_{\mathcal{H}}(G-e)+1$.

Sufficiency: Let $\gamma_{\mathcal{H}}(G-e)+1=\gamma_{\mathcal{H}}(G)$. Assume $\gamma_{\mathcal{H}}(G) \neq \gamma_{\mathcal{H}}\left(G_{e, 3}\right)$. Now by Theorem 3.1. $\gamma_{\mathcal{H}}\left(G_{e, 3}\right)=\gamma_{\mathcal{H}}(G-e)$. Applying again Theorem 3.1 we obtain $\gamma_{\mathcal{H}}(G)=\gamma_{\mathcal{H}}(G-e)-1$, a contradiction. Thus, $\gamma_{\mathcal{H}}(G)=\gamma_{\mathcal{H}}\left(G_{e, 3}\right)$. 
(ii) By (i), $\gamma_{\mathcal{H}}(G)=\gamma_{\mathcal{H}}\left(G_{e, 3}\right)$. Let $M$ be a $\gamma_{\mathcal{H}}$-set of $G-e$ and $e=u v$. By Theorem C(ii), both $u$ and $v$ are in $M$. Then (a) $M$ is a dominating $\mathcal{H}$-set of $G_{e, 1}$ and $G_{e, 2}$, (b) $M \cup\left\{x_{3}\right\}$ is a dominating $\mathcal{H}$-set of $G_{e, 4}$ and $G_{e, 5}$, and (c) $M \cup\left\{x_{3}, x_{5}\right\}$ is a dominating $\mathcal{H}$-set of $G_{e, 6}$. Hence

(A) $\gamma_{\mathcal{H}}\left(G_{e, i}\right) \leq \gamma_{\mathcal{H}}(G-e)=\gamma_{\mathcal{H}}(G)-1$ for $i=1,2 ; \gamma_{\mathcal{H}}\left(G_{e, j}\right) \leq \gamma_{\mathcal{H}}(G-e)+1=$ $\gamma_{\mathcal{H}}(G)$ for $i=4,5 ; \gamma_{\mathcal{H}}\left(G_{e, 6}\right) \leq \gamma_{\mathcal{H}}(G-e)+2=\gamma_{\mathcal{H}}(G)+1$.

By Theorem $\mathrm{C}, \min \left\{\gamma_{\mathcal{H}}(G-u), \gamma_{\mathcal{H}}(G-v)\right\} \geq \gamma_{\mathcal{H}}(G-e)$ and by Theorem A we have $\gamma_{\mathcal{H}}(G-\{u, v\})=\gamma_{\mathcal{H}}((G-u)-v) \geq \gamma_{\mathcal{H}}(G-u)-1 \geq \gamma_{\mathcal{H}}(G-e)-1$. Suppose that $\gamma_{\mathcal{H}}(G-\{u, v\})=\gamma_{\mathcal{H}}(G-e)-1$. Then both $\gamma_{\mathcal{H}}(G-u)=\gamma_{\mathcal{H}}(G-e)$ and $\gamma_{\mathcal{H}}((G-u)-v)=\gamma_{\mathcal{H}}(G-u)-1$ hold. By the second equality and Theorem A we deduce that $v$ belongs to some $\gamma_{\mathcal{H}}$-set of $G-u$. On the other hand, since $\gamma_{\mathcal{H}}(G)=\gamma_{\mathcal{H}}(G-e)+1>\gamma_{\mathcal{H}}(G-u), v$ belongs to no $\gamma_{\mathcal{H}}$-set of $G-u$, a contradiction. Thus,

(B) $\min \left\{\gamma_{\mathcal{H}}(G-u), \gamma_{\mathcal{H}}(G-v), \gamma_{\mathcal{H}}(G-\{u, v\})\right\} \geq \gamma_{\mathcal{H}}(G-e)$.

Let $D_{t}$ be a $\gamma_{\mathcal{H}}$-set of $G_{e, t}$ and $U_{t}=D_{t} \cap\left\{x_{1}, \ldots, x_{t}\right\}$, where $t=1, \ldots, 6$.

Case 1: $t \in\{1,2\}$.

Assume $U_{t} \neq \emptyset$. Then $D_{t}-U_{t}$ is a dominating $\mathcal{H}$-set for at least one of the graphs $G-e, G-u, G-v$ and $G-\{u, v\}$. Using $(B)$ we have

$$
\gamma_{\mathcal{H}}(G)=\gamma_{\mathcal{H}}(G-e)+1 \leq\left|D_{t}-U_{t}\right|+1=\gamma_{\mathcal{H}}\left(G_{e, t}\right)-\left|U_{t}\right|+1 \leq \gamma_{\mathcal{H}}\left(G_{e, t}\right)
$$

contradicting $(A)$. Thus $U_{t}$ is empty. But then $D_{t}$ is a dominating $\mathcal{H}$-set of $G-e$ which leads to $\gamma_{\mathcal{H}}\left(G_{e, t}\right) \geq \gamma_{\mathcal{H}}(G-e)$. Now by $(A)$ it follows the equality $\gamma_{\mathcal{H}}\left(G_{e, t}\right)=$ $\gamma_{\mathcal{H}}(G-e)$.

Case 2: $t \in\{4,5\}$.

Obviously $U_{t} \neq \emptyset$. As in Case 1 we obtain $\gamma_{\mathcal{H}}(G) \leq \gamma_{\mathcal{H}}\left(G_{e, t}\right)$. Since by $(A)$ $\gamma_{\mathcal{H}}\left(G_{e, t}\right) \leq \gamma_{\mathcal{H}}(G)$, we have $\gamma_{\mathcal{H}}\left(G_{e, t}\right)=\gamma_{\mathcal{H}}(G)$.

Case 3: $t=6$.

Clearly $\left|U_{6}\right| \geq 2$. As in Case 1 we obtain $\gamma_{\mathcal{H}}(G) \leq \gamma_{\mathcal{H}}\left(G_{e, 6}\right)-\left|U_{6}\right|+1$. Since $\left|U_{6}\right| \geq 2, \gamma_{\mathcal{H}}(G) \leq \gamma_{\mathcal{H}}\left(G_{e, 6}\right)-1$. Now by $(A)$ we deduce that $\gamma_{\mathcal{H}}(G)=\gamma_{\mathcal{H}}\left(G_{e, 6}\right)-1$.

(iii) Immediately by (i) and (ii). 


\section{References}

[1] D. Avella-Alaminos, M. Dettlaff, M. Lemańska, R. Zuazua, Total domination multisubdivision number of a graph, Discuss. Math. Graph Theory(accepted). http://arxiv.org/pdf/1309.7228v1.pdf

[2] M. Borowiecki, I. Broere, M. Frick, P. Mihok and G. Semanisin, A survey of hereditary properties of graphs, Discuss. Math. Graph Theory, 17(1997) 5-50

[3] E. J. Cockayne, O. Favaron, C. M. Mynhardt, On $i^{-}$-ER-critical graphs, Discrete Math. 276(2004), 111-125.

[4] M. Dettlaff, J. Raczek and J. Topp, Domination subdivision and domination multisubdivision numbers of graphs, http://arxiv.org/pdf/1310.1345v2.pdf

[5] W. Goddard, T. Haynes and D. Knisley, Hereditary domination and independence parameters, Discuss. Math. Graph Theory. 24(2004), 239-248.

[6] P.J.P. Grobler, Critical concepts in domination, independence and irredundance of graphs, Ph.D. Thesis, University of South Africa, 1998.

[7] P.J.P. Grobler, C.M. Mynhardt, Upper domination parameters and edge-critical graphs, J. Combin. Math. Combin. Comput. 33 (2000) 239-251.

[8] P. J. P. Grobler, C. M. Mynhardt, Domination parameters and edge-removal-critical graphs, Discrete Math. 231(2001), 221-239.

[9] T. W. Haynes, S. T. Hedetniemi, P. J. Slater, Domination in Graphs, Marcel Dekker, Inc., New York, NY, 1998.

[10] D. Michalak, Domination, independence and irredundance with respect to additive induced-hereditary properties, Discrete Math. 286(2004), 141-146.

[11] V. Samodivkin, Domination with respect to nondegenerate and hereditary properties, Mathematica Bohemica, 133(2008), 167-178.

[12] V. Samodivkin, Domination with respect to nondegenerate properties: bondage number, Australasian J. Combin., 45(2009), 217-226.

[13] V. Samodivkin, Domination with respect to nondegenerate properties: vertex and edge removal, Mathematica Bohemica, 138(2013), 75-85.

[14] V. Samodivkin, Upper bounds for the domination subdivision numbers of graphs on topological surfaces, Czechoslovak Mathematical Journal, 63 (138) (2013), 191-204 